\title{
The Security Quantitative Analysis Model of EED Based on Burning and Explosion Dangerous Source Evaluation Method
}

\author{
Yaohua Wang \\ Engineering Institute of Corps of Engineers, PLA Univ. of Sci. \& Tech. \\ No. 1, Haifuxiang, Nanjing 210007, China \\ Tel: 86-25-8082-0028_E-mail: qianxueningsi@yahoo.cn \\ Liang Wang (Corresponding author) \\ Engineering Institute of Corps of Engineers, PLA Univ. of Sci. \& Tech. \\ No. 1, Haifuxiang, Nanjing 210007, China \\ Tel: 86-189-360-35865 E-mail: qianxueningsi@163.com \\ Xiaoqiang Yang \\ Engineering Institute of Corps of Engineers, PLA Univ. of Sci. \& Tech. \\ No. 1, Haifuxiang, Nanjing 210007, China \\ Tel: 86-138-138-85481 E-mail: qianxueningsi@yeah.net \\ Baoguo Zhu \\ Military Representative Office of the General Department of Armaments in Tianjin Area \\ No. 7, Yuhong Road, Hebei District, Tianjin 300240, China \\ Tel: 86-133-899-10860Ｅ-mail: zhbg@163.com \\ Haishan Yang \\ Military Representative Office of the General Department of Armaments in Zhengzhou Area \\ No. 23, Eastern Rantun Road, Zhengzhou 450051, China \\ Tel: 86-139-371-96083_E-mail: yang67568578@163.com \\ Yunfeng Wang \\ Engineering Institute of Corps of Engineers, PLA Univ. of Sci. \& Tech. \\ No. 1, Haifuxiang, Nanjing 210007, China \\ Tel: 86-139-519-15575Ｅ-mail: wangxiaohan9516@126.com
}

Received: March 25, 2011

Accepted: May 17, 2011

doi:10.5539/mas.v5n4p112

The research is financed by National Great Engineering.

\begin{abstract}
In view of burning and explosion dangerous source which exist in explosive and its manufacture, the security quantitative analysis model of the emergency open system based on the burning and explosion dangerous source evaluation method is proposed, and the system security of the emergency open system is analyzed by the burning and explosion dangerous source evaluation method. The analysis results basically show the regulation of the emergency open system security. The model and the proposed research method can provide reference for security study of other aero explosive device system. The feasibility and reliability of burning and explosion dangerous source evaluation of the emergency open system have been heightened. The security quantitative analysis model of emergency open system in virtue of burning and explosion dangerous source evaluation method can be a good method of the choice of the security design of the whole system.
\end{abstract}

Keywords: System security analysis, Burning and explosion dangerous source evaluation method, Emergency open system, EED 


\section{Introduction}

To meet the aircraft flight test team members' needs for the air emergency evacuation, it is required that the probability of the false triggering of the emergency opening system of the aircraft's service door is not more than 0.001 under the premise of the confidence level can not being less than $90 \%$ when the aircraft is under normal conditions. Therefore, it is need for a security quantitative analysis of the emergency opening system.

The so-called quantitative analysis of system security, that is to analyze and determine the security problems of the emergency opening system, the probability of its possible harmful effects, and calculate the security changes taken by the appropriate safety measures in the system. By comparing the compliance status of the emergency opening system's risk before and after the security reform measures being taken, the decision-making basis is provided for selecting the best design.

The existing explosion hazard assessment has been focused on the production process effects on the security of EED (Electro-Explosive Devices), which is no need to consider in the practical application of EED. Therefore, the explosion hazard assessment method can not be fully applicable to the security analysis of the emergency opening system, new analytical methods are required to explore in the practice of specific research projects. Therefore, based on the explosion hazard assessment method, a security quantitative analysis model for the emergency opening system is put forward.

\section{Basic Structure of EED}

EED like hot bridge wire squibs, initiators, and detonators are energetic devices which are activated by application of electric current. They are first elements in an ignition train producing high temperature flames and ignite energetic materials such as pyrotechnics and booster charges. They are composed of ignition pins, bridge wire element, primary charge, and main charge which are enclosed in a metal case. The schematic structure of a typical small EED is shown in Figure 1. Electric current is applied to the ignition pins connected to the bridge wire element. Bridge wire element is a resistive thin filament which could be selected from different metals like $\mathrm{Ni}-\mathrm{Cr}$, palladium etc. With the application of electric current bridge wire element is heated due to its resistive nature. The final or equilibrium temperature of the bridge wire depends mainly on the level of current and some other physical parameters. Bridge wire element is embedded into the sensitive primary charge. The primary charge ignites when the bridge wire temperature reaches to the ignition temperature of it. Main charge exists in front of the primary charge and sometimes they are separated with a thin celluloid film. Flame originating from the primary charge ignites the main charge and consequently EED operates, producing hot and pressurized gas with flames. (Suzan Koc, H. Tugrul Tinaztepe, 2006, pp.415-426).

\section{The explosion hazard assessment method and its simplified}

The explosion hazard assessment method is a risk source assessment method suitable for the production and storage business of EED, it is based on the safety system engineering principles, combined with the explosion hazard and safety management experience of military industry and civil explosive business, and the essence of hazard assessment from abroad and other industries, it is a pioneering creation of the comprehensive security of EED. The real properties of assessment is the calculation of risk of the system itself by the explosive risk coefficient $\alpha$, the explosive dosage and power coefficient $\beta$ in the system, technology risk coefficient $\gamma$, system security risk factor $K$, and the frequency index $D$ that personnel exposure to hazards within a safe distance, calculate the influence on the outside of the system according to safety distance by accidents. The evaluation model is as follows,

$$
H=H_{\text {in }}+H_{\text {out }}
$$

The $H$ in above equation indicates the inherent danger still existing in the system after effective control of the degree of risk, and it also indicates the severity and possibility of dangers. The risk value is relative, not absolute, and the risk of the system can be reflected only in the comparison of similar hazards. A relatively independent unit of the risk of combustion and explosion is taken as the research object generally, such as the electro-explosive system, door pushing power devices and explosive cutting cords used in the emergency opening system and so on.

$H_{\text {in }}, H_{\text {out }}$ indicates the internal, external harm degree of system respectively, in the event of an accident of explosion hazard. Which

$$
\begin{gathered}
H_{\text {内 }}=\alpha \beta+K \alpha \beta \gamma D \\
H_{\text {外 }}=\sum\left(1-\frac{R_{1 i}}{R_{0 i}}\right) \cdot C_{i}
\end{gathered}
$$

According to the actual situation of the aviation environment in which the emergency opening system, the explosion hazard assessment model is simplified and distorted. The design safe distance of the emergency opening system is $1.5 \mathrm{~m}, 12$ crew members are $3 \mathrm{~m}$ away of the system when test flight. The crew will not be 
exposed to the system within the safe distance when test flight, it will not has any damage and impact on the outside personnel and equipment of system away the safe distance, in the event of the occurrence of an accidental explosion. Therefore, when the explosion hazard assessment model is simplified, the hazard outside of the system $H_{\text {out }}$ is abandoned, the indicator $D$ of the frequency of personnel exposure to hazards within the safe distance is not considered. This time

$$
H=H_{\text {in }}=\alpha \beta+K \alpha \beta \gamma
$$

The technology risk coefficient $\gamma$ in above equation indicates a sensitivity characteristics factor in the manufacture processes of explosives and their products. They are the key points of consideration in the production and processing, and in the emergency opening system, the explosive used is the qualified products of a certain safety factor, the impact of these sensitivities can not be considered, so it is gave up to analyze this parameter in the process of simplifying the explosion hazard evaluation method. This time

$$
H=\alpha \beta+K \alpha \beta
$$

The risk coefficient $\alpha$ in above equation may be subject to the external form of energy in the production process, and it is a characteristic coefficient of sensitivity. The stability of explosive and its products is only relative, temporary, and when it was the role of external energy, it can be excited and dramatic release of energy combustion or explosion of chemical reactions. The difficulty of this situation is usually described by the sensitivity. The higher the sensitivity, the more prone to fire or explosion, it is slightly excited by the outside role. Clearly, the sensitivity can occur as a measure of the possibility of combustion explosion. Explosives and their products in the production and processing may be the role of energy in the form of outside, hot, fire, mechanical (impact, friction), electrostatic and electromagnetic radiation effects, and sometimes may also be generated by other explosive shock wave. So there is the corresponding thermal sensitivity, flame sensitivity, mechanical sensitivity, electrostatic sensitivity, electromagnetic radiation sensitivity and detonation shock sensitivity, etc. (Guoshun Zhang, 2006, pp.31-31). According to hazard identification and accident causation theory, the properties risk coefficient $\alpha$ is closely related to the expected probability index $P$ of false triggering of the explosive in the system, so it is suitable to replace the properties risk coefficient $\alpha$ using $P$ in the process of simplifying explosion hazard assessment method. This time

$$
H=P \beta+K P \beta
$$

Finally the security quantitative analysis model for the emergency opening system is gotten as follows,

$$
H=P \beta+K P \beta=P \beta(1+K)
$$

\section{Model Application}

\subsection{Parameters determination}

\subsubsection{The expected probability index value $P$ of false triggering of the explosive in the system}

We establish a fault tree to have a quantitative analysis of the false triggering accident of the aircraft emergency opening system, based on false trigger probability of basic events, by solving the calculation, when the aircraft is under normal conditions, the top event probability of false triggering is 0.000998 under the premise of the confidence level not less than $90 \%$. Comparing the corresponding relationship between the expected probability value $P$ of the special state and the risk probability of various states, as the reference table 1 shows that the event is the situation of "an extremely unlikely event of accident", we get the expected probability index value $P=1$ of false triggering of the explosive in the system. (Yaling Shang, etl, 2009, pp.66-70).

\subsubsection{The explosive dosage and power coefficient $\beta$ in the system}

The explosive dosage and power coefficient $\beta$ in the system reflects the destructive power of the system's explosive combustion or explosion, and the severity of a risk.

When the fire or explosion of the explosive occurred, causing the damaging effects on airborne equipment, there are vibration and shock, combustion and thermal radiation, explosion fragments, blast pressure and noise. Known by the explosion theory,

$$
\beta=\sqrt[3]{M \cdot Q / Q_{T N T}}
$$

Where $M$ - The system explosive dosage;

$Q$ - The explosive heat of the EED used in the system, $J \cdot \mathrm{kg}^{-1}$;

$Q_{T N T}$ - TNT explosive heat, $J \cdot \mathrm{kg}^{-1}$;

$Q / Q_{T N T}$ - TNT explosive equivalent. 
The main charge of explosion-style door pushing power devices used in the emergency opening system is black powder, its density is $1.8 \mathrm{~g} / \mathrm{cm}^{3}$, dosage is $52 \mathrm{~g}$.

The main charge of the lock, hinge plate and the skin cutting cord, and silver detonating cord, blasting caps, explosive expansion device is RDX, the line density of the lock, hinge plate cutting cord is $18.5 \mathrm{~g} / \mathrm{m}$, the dosage is $22 \mathrm{~g}$; the line density of the skin cutting cord is $2.2 \mathrm{~g} / \mathrm{m}$, the dosage is $4 \mathrm{~g}$; the linear density of silver detonating cord is $0.06 \mathrm{~g} / \mathrm{m}$, the dosage is $0.78 \mathrm{~g}$, the charge capacity of 3 detonators is $0.22 \mathrm{~g}$; the charge capacity of 18 explosive expansion device is $1.8 \mathrm{~g}$. The RDX explosive of total consumption in the system is $22+4+0.78+0.22$ $+1.8=28.8 \mathrm{~g}$.

The dosage of the boron/potassium nitrate used for ignition is $0.4 \mathrm{~g}$.

TNT explosive equivalent: black powder is $0.45, \mathrm{RDX}$ is 1.39 , and boron/potassium nitrate is 0.87 . (Jianhong Yang, etl, 2006, pp.299-301). (Jianhong Chen, etl, 2003, pp.79-80).

Thus available, the explosive dosage and power coefficient $\beta$ of black powder in the system is:

$$
\beta_{1}=\sqrt[3]{52 \times 0.45} \approx 2.86
$$

The explosive dosage and power coefficient $\beta$ of $\mathrm{RDX}$ in the system is:

$$
\beta_{2}=\sqrt[3]{28.8 \times 1.39} \approx 3.421
$$

The explosive dosage and power coefficient $\beta$ of boron/potassium nitrate in the system is:

$$
\beta_{3}=\sqrt[3]{0.4 \times 0.87} \approx 0.703
$$

The obtained explosive dosage and power coefficient $\beta$ in the system is:

$$
\beta=\beta_{1}+\beta_{2}+\beta_{3}=2.86+3.421+0.703=6.984
$$

\subsubsection{The system security risk factor $K$}

The system security risk factor $K$ is the degree of security risks that the risk factors should have been effectively controlled by the system, the risk factors of the system that can pose security risks is extremely complex. Based on three-ring accident causation theory, the security risk factor model is as follows, (Guoshun Zhang, 2006, pp.160-160).

$$
K=6.1 \Omega_{P} \Omega_{M}+2.2 \Omega_{P} \Omega_{E}+1.7 \Omega_{M} \Omega_{E}
$$

According to the three-ring accident causation theory, the potential dangers of the system show into accidents because of "unsafe acts of workers", "machine (physical) state of insecurity" and "environment unsafe conditions". It is triggered by that two-two meet in the same time and space. 6.1, 2.2 and 1.7, indicates the weight coefficient respectively, which are based on a large number of accidents statistics, accident by the two-two intersection of the three subsystems of the "man - machine (object)", "man - environment" and "machine (object) - Environment".

If we use $S_{\mathrm{P}}, S_{\mathrm{M}}, S_{\mathrm{E}}$ respectively indicate the safety standards of man, machine (materials), environmental values should meeting, $S_{\mathrm{x}}, S_{\mathrm{Y}}, S_{\mathrm{Z}}$ respectively indicate the real security status value of man, machine (materials), environmental, then $S_{\mathrm{X}} / S_{\mathrm{P}}, S_{\mathrm{Y}} / S_{\mathrm{M}}, S_{\mathrm{Z}} / S_{\mathrm{E}}$ indicates the man, machine (materials), environmental safety compliance rate, so there is that $\left(1-S_{\mathrm{X}} / S_{\mathrm{P}}\right)$ indicates the "unsafe acts $\Omega_{\mathrm{P}}$ of workers", $\left(1-S_{\mathrm{Y}} / S_{\mathrm{M}}\right)$ indicates the "machine (physical) state $\Omega_{\mathrm{M}}$ of insecurity", $\left(1-S_{\mathrm{Z}} / S_{\mathrm{E}}\right)$ indicates the "environment unsafe conditions $\Omega_{\mathrm{E}}$ ". Eventually, the system security risk factor model is expressed as:

$$
K=6.1\left(1-S_{X} / S_{P}\right)\left(1-S_{Y} / S_{M}\right)+2.2\left(1-S_{X} / S_{P}\right)\left(1-S_{Z} / S_{E}\right)+1.7\left(1-S_{Y} / S_{M}\right)\left(1-S_{Z} / S_{E}\right)
$$

Through the detailed analysis of previous test data in the system development process, combined with expert judgments method, the safety compliance rates of $S_{\mathrm{X}} / S_{\mathrm{P}}, S_{\mathrm{Y}} / S_{\mathrm{M}}, S_{\mathrm{Z}} / S_{\mathrm{E}}$ respectively are $0.9999,0.99999,0.90$, so $\left(1-S_{\mathrm{X}} / S_{\mathrm{P}}\right)=0.0001, \quad\left(1-S_{\mathrm{Y}} / S_{\mathrm{M}}\right)=0.00001, \quad\left(1-S_{\mathrm{Z}} / S_{\mathrm{E}}\right)=0.1, \quad$ the system security risk factor $K=6.1 \times(0.0001 \times 0.00001)+2.2 \times(0.0001 \times 0.1)+1.7(0.00001 \times 0.1)=2.37061 \times 10^{-5}$ after taking them into the equation (4).

Bring the results of the above calculations into the equation (1), and we can get the emergency opening system risk $H=P \beta(1+K)=1 \times 6.984 \times\left(1+2.37061 \times 10^{-5}\right)=6.9841655634024 \approx 6.98$ of the aircraft under normal conditions.

\subsection{The transformation model based on the system}

According to the new model, calculate the risk of explosive within the system, the main risk factors increased is analyzed, the optimization safety measures and corrective measures is proposed to reduce the risk degree of explosive in the system, in order to achieve the risk indicator of system design requirements. The risk indicator value of explosive of the emergency opening system is required to below 6 , and while the risk indicator of the initial system development is 6.98 , the safety design requirements to the system can not be met, and it is need to take safety measures for the system transformation. 
On the premise of meeting the requirements to system functions, the explosive structure design and charge way of the emergency opening system is improved, the black powder dosage used in the door pushing power devices is reduced to $18 \mathrm{~g}$.

The line density of the lock, hinge plate cutting cord is reduced from $18.5 \mathrm{~g} / \mathrm{m}$ to $13.5 \mathrm{~g} / \mathrm{m}$, the dosage reduces to $4.92 \mathrm{~g}$; the line density of the skin cutting cord is reduced from $2.2 \mathrm{~g} / \mathrm{m}$ to $1.8 \mathrm{~g} / \mathrm{m}$, the dosage reduces to $3.744 \mathrm{~g}$; the dosage of the silver detonating cord, detonators and explosive expansion device remains $0.78 \mathrm{~g}, 0.22 \mathrm{~g}$ and $1.8 \mathrm{~g}$. Thus the dosage of RDX explosives consumed in the system drops to $4.92+3.744+0.78+0.22+1.8=$ $11.464 \mathrm{~g}$.

The dosage of the boron/potassium nitrate used for ignition is $0.4 \mathrm{~g}$ still.

Thus, the improved explosive dosage and power coefficient $\beta$ in the system is:

$$
\beta^{\prime}=\beta_{1}^{\prime}+\beta_{2}^{\prime}+\beta_{3}=\sqrt[3]{18 \times 0.45}+\sqrt[3]{11.464 \times 1.39}+0.703 \approx 2.0+2.516+0.703=5.219
$$

Through the implementation of anti-vibration system, anti-high temperature, thermal shock resistance, anti-electromagnetic radiation, anti-static and other security reinforcement measures, the security standard rate $S_{\mathrm{Y}} / S_{\mathrm{M}}$ of machine (physical) increases to 0.999995 , so $\left(1-S_{\mathrm{Y}} / S_{\mathrm{M}}\right)=0.000005$, the system security risk factor $K^{\prime}=6.1 \times(0.0001 \times 0.000005)+2.2 \times(0.0001 \times 0.1)+1.7 \times(0.000005 \times 0.1)=2.285335 \times 10^{-5}$ is got by substituting them into equation.

The expected probability index value $P$ of false triggering of the explosive in the system is 1 still.

Bring the improved results of the above parameters into the equation (1), we get the improved system risk indicator $H^{\prime}=1 \times 5.219 \times\left(1+2.28535 \times 10^{-5}\right)=5.21911927163365 \approx 5.22$.

This shows that by taking safety transformation measures, the risk of explosive of the emergency opening system drops to 5.22 from 6.98 , the risk indicators of the design requirements is achieved to determine the feasibility of the design.

\section{Conclusions}

The security quantitative analysis model for the emergency opening system based on explosion hazard assessment method is established. It is considered in the model that the emergency opening system itself and the characteristics of its aviation environment, and the existing assessment model is simplified and distorted reasonably. The system $H_{\text {out }}$ to the outside world outside the abandoned hazard, personnel exposure to hazards the frequency of the safety distance will not be considered indicators of $D$ to discard the coefficient $\gamma$ on the process hazard analysis, the probability of false triggering by index value is expected to replace the physical properties of $P$ risk coefficient $\alpha$. The hazard outside of the system $H_{\text {out }}$ is abandoned, the indicator $D$ of the frequency of personnel exposure to hazards within the safe distance is not considered, The technology risk coefficient $\gamma$ is gave up to analyze, the properties risk coefficient $\alpha$ is replaced by the expected probability index $P$ of false triggering of the explosive in the system. The re-establish model is analyzed from the two aspects of the accident consequences severity and accident probability, it is facilitative and simple to access parameters and calculate in the re-establish model. Adopt the model to calculate the compliance status of the emergency opening system's risk before and after the security reform measures being taken, and it is a good consistency with the actual situation. Thus, a quantitative analysis method is provided for the choice of system security design by this model.

After shock and vibration test, high and low temperature test, thermal shock testing, environmental testing of electromagnetic static, and the real test environment, the test of practice, the emergency opening system security to the design requirements are achieved, the practical value of the model in the emergency opening system security quantitative analysis is affirmed, a reference for a similar system security quantitative analysis is provided.

It can be seen from the model, system security involve the probability and severity of consequences of the accident. The accident probability is affected cooperatively by the whole safety of the emergency opening system, the accident prevention measures and its effectiveness. The loss of personnel and equipment is determined by the size of the energy released of the accident, which also directly determine the severity of the consequences of the accident. Therefore, whether to reduce the probability of occurrence of the accident, or in order to reduce the severity of consequences of the accident, are required to implement the exact amount of explosives.

\section{References}

Baohui Jia, Lei Gao, Yidong Du. (2010). Aircraft Air Conditioning System Security Analysis Based on FTA. Aviation Maintenance and Engineering, 3, 74-76.

Bao Guo. (1998). Safety Analysis Method and Applications of Civil Aircraft Systems. Xifei Technology, 26, 72-81.

Guoshun Zhang. (2006). Explosion Hazards and Safety Technology. Beijing: China Electric Power Press, 31-31, 
160-160.

Jianhua Geng, Xinshe Song. (2002). Preliminary Study on Explosive Hazard Evaluation in Assembly Workshop. Explosive, 2, 46-50.

Jianhong Chen, Xiaodong Su. (2003). Experimental Value Study of Black Powder's TNT Equivalent. Criminal Technology, 29, 79-80.

Jianhong Yang, Jinglong Xu. (2006). Calculation of Propellants TNT Equivalent. Computer Simulation, 23: 299-301.

Keqing Cui. (2005). Theory and Technology of Combustion and Explosion in Safety Engineering. Beijing: China Metrology Press, 377-390.

MIL-STD-1576. Electro explosive Subsystem Safety Requirements and Test Methods for Space Systems,52-54.

Mark W. Heyse, Keith A. Jamison, Ronald E. Stearns. (2006). Design, testing, and safety considerations of an Optically Isolated Firing Set. Air Force Research Laboratory Munitions Directorate,298-312.

Suzan Koc, H. Tugrul Tinaztepe. (2006). A Study for the Effect of Low Level Conducted Periodic Pulsed Currents and Electromagnetic Environment on EED Systems. 42nd AIAA/ASME/SAE/ASEE Joint Propulsion Conference \& Exhibit, 415-426.

W. E. Baker and others. Guoshun Zhang etl. translate. (1988). Explosion Hazards and Assessment. Beijing: Mass Press, 82-84.

Wenyao Huang, Shilong Yan. (2009). Explosive Chemistry and Manufacture. Beijing: Metallurgical Industry Press, 37-47.

X.-Y. Wu, Q. Liu. (2008). Equipment Test and Evaluation. Beijing: National Defense Industry Press, 56-58.

Yaling Shang, Jinhuang Wu, Zhibiao Lv. (2009). Explosion Hazard Simplified Quantitative Assessment Method in the Maintenance and Use. Detection \& Control, 31, 66-70.

Yanlei Qin, Yushi Lu, Juan Wang, et al. (2006). Comparison Study of System Safety Analysis Method. Journal of Safety Science and Technology, 2, 69-78.

Table 1 . The corresponding relationship between the expected probability value $P$ of the special state and the risk probability of various states

\begin{tabular}{c|c}
\hline Index value $P$ & The case of an expected accident \\
\hline 10 & the accident is entirely to be expected \\
8 & the likelihood of accidents is very large \\
6 & may occur, but not often \\
4 & may occur, completely unexpected \\
3 & $\begin{array}{c}\text { Me accident possibility can be envisaged } \\
\text { very unlikely event of an accident } \\
\text { a very unlikely event of an accident } \\
\text { almost no such accident }\end{array}$ \\
\hline 1 & $\begin{array}{c}\text { Mitigated } \\
\text { outlet wall } \\
\text { Case } \\
\text { Main charge }\end{array}$ \\
\hline
\end{tabular}

Figure 1. Basic structure of EED 